\title{
ADNP prompts the cisplatin-resistance of bladder cancer via TGF- $\beta$-mediated epithelial-mesenchymal transition (EMT) pathway
}

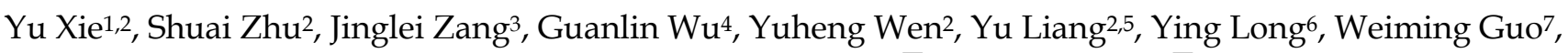 \\ Chuanbing Zang8, Xiang Hu${ }^{1}$, Gang Fan ${ }^{2,9}$, Shuanglin Xiang ${ }^{1 凶}$ and Jian Zhang ${ }^{\bowtie}$ \\ 1. Key Laboratory of Protein Chemistry and Developmental Biology of the Ministry of Education, Hunan Normal University, 410081 Changsha, China. \\ 2. Department of Urology, the Affiliated Cancer Hospital of Xiangya School of Medicine, Central South University, Hunan Cancer Hospital, 410013 Changsha, \\ China. \\ 3. Changsha Health Vocational College, 410600 Changsha, China. \\ 4. Department of Pathology, School of Basic Medical Sciences, Fudan University, 200433 Shanghai, China. \\ 5. Pingxiang Maternal and Child Care Hospital, 337000 Pingxiang, China. \\ 6. Clinical Translational Research Center, the Affiliated Cancer Hospital of Xiangya School of Medicine, Central South University, Hunan Cancer Hospital, \\ 410013 Changsha, China. \\ 7. The 2nd Affiliated Hospital of South China University, 421001 Hengyang, China. \\ 8. Medizinische Klinik m. S. Hämatologie u. Onkologie, Campus Bejamin Franklin, Unviersitätsmedizin Berlin Charité, 12203 Berlin, Germany. \\ 9. Department of Urology, Huazhong University of Science and Technology Union Shenzhen Hospital; the 6th Affiliated Hospital of Shenzhen University \\ Health Science Center, 518060 Shenzhen, China.
}

$\triangle$ Corresponding authors: Prof. Dr. Jian Zhang, Key Laboratory of Protein Chemistry and Developmental Biology of the Ministry of Education, Hunan Normal University, Yuelunan Road 168, Yuelu, Changsha. E-mail: hnc_uro@yeah.net; Prof. Dr. Shuanglin Xiang, Key Laboratory of Protein Chemistry and Developmental Biology of the Ministry of Education, Hunan Normal University, Yuelunan Road 168, Yuelu, Changsha. E-mail: 1715840770@qq.com.

(c) The author(s). This is an open access article distributed under the terms of the Creative Commons Attribution License (https://creativecommons.org/licenses/by/4.0/). See http://ivyspring.com/terms for full terms and conditions.

Received: 2021.01.11; Accepted: 2021.05.19; Published: 2021.06.22

\begin{abstract}
Activity-dependent neuroprotective protein (ADNP) is vital for embryonic development and brain formation. Besides, the upregulated expression of ADNP enhances tumorigenesis in some human tumors like bladder cancer $(B C)$. However, the potential roles of ADNP in drug resistance and the related mechanisms in $B C$ is unknown. We performed this study to elucidate the influence of ADNP in the chemoresistance of $\mathrm{BC}$ and tried to explore the underlying molecular mechanism. The expressions of $A D N P$ in $B C$ from progression and non-progression patient specimens were measured by quantitative real-time PCR ( $\mathrm{QRT}-\mathrm{PCR}$ ) and immunohistochemistry (IHC). In vitro experiments including colony formation, cell counting kit-8 (CCK-8), wound healing, and in vivo tumorigenesis assay were performed to explore the effects of ADNP on chemoresistance of BC. The impacts of ADNP on TGF- $\beta /$ Smad signaling pathways were explored by western blot. Our results showed that the expression of ADNP mRNA and protein were significantly upregulated in $B C$ tissues of the patients who suffered tumor-progression via RT-PCR and western blot. Cox regression survival analysis revealed that patients with high ADNP expression closely linked to shorter tumor-free survival. ADNP downregulation in BC showed more sensitive to cisplatin in vivo, while ADNP overexpression showed the opposite results. Additionally, we confirmed that ADNP promoted cell migration and EMT, thereby inducing cisplatin resistance, which may be related to TGF- $\beta$ / Smad signaling pathway.
\end{abstract}

Key words: ADNP; bladder cancer; epithelial-mesenchymal transition; chemoresistance; TGF- $\beta /$ Smad

\section{Introduction}

Bladder cancer $(\mathrm{BC})$ is one of the most common malignancies of the human urinary system [1]. Chemotherapy remains a vital therapeutic strategy for BC $[2,3]$. Despite significant advances in BC research in the past few decades, the survival of patients with
BC still faced severe challenges [4]. So there is urgent need to explore the underlying mechanisms of chemoresistance in BC. Previous studies indicated a molecular and phenotypic association between chemoresistance and the acquisition of the epithelial- 
mesenchymal transition (EMT)-like phenotype in many types of cancers such as breast cancer, glioblastoma, hepatocellular carcinoma [5-9]. For example, EMT involved in chemoresistance of cancer cells against conventional therapeutics including cisplatin, vincristine and oxaliplatin [10]. On the mechanism, TGF $\beta$ signaling was associated with chemoresistance and EMT characteristics, since TGF $\beta$-induced EMT increased chemoresistance in hepatocellular carcinoma.

Activity-dependent neuroprotective protein (ADNP) is a protein that plays a crucial role in neuroprotective responses to cellular growth and proliferation [11]. ADNP was frequently amplified and overexpressed in many human malignancies, such as ovarian [12]. ADNP second cDNA clone (H3) differed from clone $\mathrm{H} 7$ in several polymorphic regions and polyadenylation sites. In addition, clone H3 contains frameshift mutations with premature termination codon at position 3408 [13]. The discovery of ADNP mutations in neurological disorders ignited an interest to explore the role of ADNP in cancer. Studies have shown that under different stress conditions, ADNP may mutate or differ in expression, thus accelerating the progression of cancer [14]. Wild type ADNP can impact cellular proliferation via the WNT signaling pathway in colon carcinoma [15]. However, the activation of ADNP signaling system, mediated by an endogenous pituitary adenylate cyclase-activating polypeptide, can increase the resistance of malignant peripheral nerve sheath tumor to $\mathrm{H}_{2} \mathrm{O}_{2}$-induced death with serum starvation [16]. Our previous study has shown that overexpression of ADNP in BC stimulated the Akt-MDM2-p53 pathway and enhanced binding of cyclin D1 to CDK4 or CDK6, accelerating the cell cycle transition from G1 phase to $S$ phase [17]. ADNP may act as an oncogene in certain cellular contexts. Hence, we speculate that ADNP contribute to chemoresistance of $\mathrm{BC}$ through modulating EMT processes. In this study, we preliminarily investigated the relationship between chemoresistance of ADNP and BC, and the effect of ADNP on TGF- $\beta$ signaling pathway of EMT.

\section{Materials and Methods}

\section{Tissue samples and cell lines}

All patients had non-muscle invasive bladder migration. The clinical stages included CIS, Ta and T1. Before inclusion, all papillary tumors were completely removed. Patients with T1 disease underwent urethrectomy. The included patients had sufficient bone marrow function (more han 1500 blood granulocytes $/ \mathrm{mm}^{3}$ and more than 150,000 platelets $\left./ \mathrm{mm}^{3}\right)$. Patients were $>18$ years old and had the ability to provide informed consent. Immunosuppressed patients (for example, use of HIV, chronic steroids) were excluded.

All of paraffin-embedded specimens of human non-muscle invasive bladder transitional cell carcinoma tissues were obtained from the patients who were recruited in the Affiliated Cancer Hospital of Xiangya Medical College of Central South University from January 2015 to December 2017. Another 43 non-muscle invasive bladder urothelial carcinoma surgical fresh specimens were also collected randomly, including 25 patients with progression $\mathrm{BC}$ and 18 patients with non-progression BC. All patients were diagnosed by histopathology and were treated with bladder tumor resection and intravesical chemotherapy. We defined non-progression BC by using urethrocystoscopy, CT, and urinary shedding, indicating no bladder tumor progression during 3-year follow-up. By 3-year follow-up biopsy, a mature invasive transitional cell carcinoma of the lamina propria is defined as progression $\mathrm{BC}$.

Human BC cell lines, including T24, BIU87, 5637, and TCCSUP, were obtained from Sun Yat-sen University Cancer Center (Guangzhou), and cultured in RPMI-1640 medium (Gibco, USA) and 10\% fetal bovine serum (Gibco, USA) + 1\% Streptomycin and placed in a $5 \% \mathrm{CO} 2,37^{\circ} \mathrm{C}$. Prior to the use of these clinical data for research, informed patient consent and approval from the institutional research ethics committee were obtained.

The study was approved by the ethics committee of the Affiliated Cancer Hospital of Xiangya School of Medicine, Central South University (No: 2016-0008). This study was conducted in accordance with the following ethical guidelines: the Helsinki Declaration, the International Code of Ethics in Biomedical Research (CIOMS) involving human subjects, the Belmont Report, and the American Common Rules.

\section{Quantitative real-time PCR (qRT-PCR)}

mRNA were measured as previously described [18-20]. Total RNA was extracted from the tissues using TRIzol reagent (Invitrogen, USA) according to the manufacturer's instructions. Spectrophotometry (A260 / A280 = 1.8-2.0) was used to determine RNA purity, and cDNAs were generated with M-MLV transcriptase according to the manufacturer's instructions (BioRAD, USA). The quantitative realtime PCR mixture system is $1 \mu \mathrm{g}$ cDNA, $0.4 \mathrm{ul}$ of the target gene primer, and $5 \mathrm{ul} 2 \times$ SYBR Green (BioRAD, USA). The reaction was performed using the LightCycler480 Real-Time PCR System (BioRAD, USA). The reaction procedure is: $1 \mathrm{~min}$ at $95^{\circ} \mathrm{C}$, followed by 35 cycles of $95^{\circ} \mathrm{C}$ for 15 seconds, $95^{\circ} \mathrm{C}$ for 
10 seconds, $65^{\circ} \mathrm{C}$ for 60 seconds, and finally $97^{\circ} \mathrm{C}$ for 1 minute. The $2^{-\triangle \Delta C T}$ method was used to determine the relative gene expression level of non-progression BC tissue and progression BC tissue. ADNP amplification primer sequences are:

\section{- Forward: 5'-CATCCTGCGTCTGGACCTGG-3';}

- Reverse: 5'-TAATGTCACGCACGATTTCC-3'.

\section{Western blot analysis}

Western blot analysis was performed as previously described [21], tissues and cells were washed with PBS, and lysed with RIPA lysis buffer including protease lysis inhibitor (Roche USA) and phosphatase inhibitor (Roche USA). A BCA protein assay kit (Thermo Scientific, USA) was used to measure protein concentration. 30ug protein extract was separated with a $10 \%$ SDS-polyacrylamide gel and transferred to a PVDF membrane (microwell). The membrane was blocked with TBST buffer containing $5 \%$ skim milk at $37{ }^{\circ} \mathrm{C}$ for 2 hours, incubated with primary antibody at $4{ }^{\circ} \mathrm{C}$ overnight, and incubated with peroxidase-conjugated secondary antibody for 2 hours. The signal was detected using ECL kit (Cell Signaling Technology, 12757) and photographed. Bands were quantified using grayscale analysis software (Bio-Rad). GAPDH expression is used as a loading control to standardize the expression of other proteins. The main antibodies are as follows: anti-ADNP (1: 1000, Proteintech, USA), anti-GAPDH, N-Cadherin, Vimentin, Snail, E-Cadherin, $\beta$ - Catenin, Claudin- 1 , TGF- $\beta$, TGF- $\beta$ R1, Smad2/3, p-Smad2/3 (1: 1000, Cell Signaling Technology, USA). The secondary antibodies are as follows: HRP-goat anti-rabbit Ig G, HRP-goat antimouse Ig G (1: 5000, Cell Signaling Technology, USA).

\section{Immunostaining}

Immunostaining was performed as previously described [22, 23], the paraffin-embedded tissue was cut into $5 \mathrm{~mm}$ slices, and baked at $60{ }^{\circ} \mathrm{C}$ for $2 \mathrm{~h}$. The slices dewaxed in xylene and hydrated in gradient ethanol. Endogenous enzymes were removed in $0.3 \%$ hydrogen peroxide. The slices were added into EDTA antigen repair buffer and heated. The first antibody was added for incubation (ADNP, E-Cadherin, NCadherin and Vimentin (1: 1000, Abcam ab199120) at $4{ }^{\circ} \mathrm{C}$ overnight. Slices were washed by PBS. Slices were incubated with HRP-conjugated goat -rabbit or goat-mouse secondary antibody at $37{ }^{\circ} \mathrm{C}$. DAB is applied to color rendering. After the slices were dehydrated and transparent, they were sealed with neutral gum.

The immunostaining degree of paraffin embedded sections was scored independently by two experienced pathologists. Both of them were blind to the histopathological characteristics and clinical data of the specimens. All fields of a slice were analyzed. The score was based on the proportion and intensity of positive staining of tumor cells. The percentage score of tumor cells was as follows: 0: no positive tumor cells; 1 : $<10 \%$ tumor cell positive; 2 : $10-35 \%$ positive tumor cells; $3: 36-75 \%$ positive tumor cells); 4. $75 \%$ positive tumor cells. The staining intensity was graded according to the following standards: 1.no staining; 2. Weak staining (light yellow); 3. Moderate staining (light brown); 4 . Strong staining (brown). The staining index is calculated as the product of the proportion of positive tumor cells and the staining intensity score. The staining index $(0,1,2,3,4,6,8,9$, 12) was used to evaluate the expression of ADNP in BC. Staining index score $\geq 6$ was defined as high expression, while score $<6$ was defined as low expression [24].

\section{Cell transfection}

ADNP overexpression plasmid (ViGene, Shandong) and empty vector (ViGene, Shandong, China) were used. The ADNP expression construct was generated by cloning the full-length human ADNP cDNA into a pMSCV-retro-puro vector (Clontech, Palo Alto, CA). For ADNP knockdown, the trispecific knockdown site ADNP-shRNA (KD-1: CAACATGACTGATGGAGTA; KD-2: GCAAATGCC TCTACTGTAA; KD-3: TAGTAAGACTGCTGAC AAA) and negative control shRNA (NC) were purchased from Shanghai Jikai, China Biotechnology. Cells were transfected with lentivirus when cells reached $30-50 \%$ confluency. Transfected cells were screened with $1 \mathrm{ug} / \mathrm{ml}$ puromycin (Sigma-Aldrich) after $72 \mathrm{~h}$. The stable transfected cell line was maintained in a culture medium containing puromycin at $0.1 \mathrm{ug} / \mathrm{ml}$.

\section{Cell viability assay}

Cell viability were measured as previously described [25, 26]. Approximately 4000 stably transfected cells were seeded on 96-well plates. The cells were treated with different concentrations of cisplatin for $24 \mathrm{~h}$ after adhered, $10 \mathrm{ul}$ of Cell Counting Kit-8 reagent (CCK8, Dojindo Kumamoto, Japan) was added into each well and incubated for $4 \mathrm{~h}$. The absorbance was measured with a spectrophotometer (Molecular Devices, Sunnyvale, CA, USA) at a wavelength of $450 \mathrm{~nm}$.

\section{Colony growth assay}

Approximately 3000 stably transfected cells were seeded on 6 well plates and cultured for 15 days. The medium was changed every 3 days. Cells were washed twice with PBS before being harvested, 
stained with crystal violet and photographed [27, 28]. The stained colonies were counted with imageJ.

\section{Wound healing experiment}

Stably transfected cells $\left(1 \times 10^{6}\right)$ were cultured in 6 well plates until there was $60 \%-70 \%$ confluence. The scratch was made with a sterile pipette tip $(10 \mu \mathrm{l})$, and the exfoliated cell was washed away with PBS. The images were obtained under the microscope and the wound distance was recorded and calculated with Image $J$ at $0 \mathrm{~h}$ and $24 \mathrm{~h}$, respectively. The migration percentage was calculated according to the following formula: Healing rate $=[(0 \mathrm{~h}$ distance $-24 \mathrm{~h}$ distance) $/ 0 \mathrm{~h}$ distance $] \times 100 \%[29,30]$.

\section{Tumor xenograft experiments}

As previously described [31, 32], 4-week-old NOD/SCID nude mice were purchased from Hunan SJA Experimental Animal Company (Changsha, Hunan). Stable ADNP knockdown T24 cells and negative control T24 cells $\left(1 \times 10^{6}\right.$ cells) were injected into the right armpit skin of nude mice ( 5 per group), and each nude mouse in the drug group were injected with $5 \mathrm{mg} / \mathrm{kg}$ of cisplatin once every two days from the day $7^{\text {th }}$ after injection. Control group were injected with equivalent PBS. The tumor length (L) and width (W) were measured with a vernier caliper every 5 days. The volume of the tumor was calculated according to the following formula: Volume= $\left(L \times W^{2}\right) / 2$. Tumor weight was measured at the end of the experiment. Animals were sacrificed on the day 42nd. Tumors were isolated, weighed and immunostained with anti-ADNP, anti-N-cadherin, anti-E-cadherin and anti-Vimentin. The animal feeding and experimental operations were performed under SPF conditions and were approved by the Animal Ethics Committee of the Affiliated Cancer Hospital of Xiangya Medical College of Central South University. The study was approved by the animal ethics committee of the Affiliated Cancer Hospital of Xiangya School of Medicine, Central South University (No: 2019-007).

\section{Statistical analysis}

All statistical analyses were performed using the SPSS 21.0 statistical software (SPSS Incorporated, Chicago). All data were from at least three independent repeated experiments. Mean \pm SD significant tests were used, and the specific test was performed by the student test. Chi-square test was used to analyze the relationship between ADNP expression and clinicopathological characteristics. Survival curves were drawn using Kaplan-Meier method and compared by log-rank test. Survival data were analyzed using univariate and multivariate cox-regression. All cases were statistically significant with $\mathrm{P}<0.05$.

\section{Results}

\section{ADNP was up-regulated in patients suffered tumor-progression}

We randomly collected the surgical fresh specimens from $43 \mathrm{BC}$ patients, including 25 patients with tumor-progression and 18 patients with no-progression. The expression of ADNP mRNA was up-regulated in the patients who suffered tumorprogression versus in the patients with no-progression $(P<0.05$, Fig. $1 \mathrm{~A})$. Additionally, the immunohistochemistry of $128 \mathrm{BC}$ tissues showed that ADNP protein was also significantly overexpressed in patients with tumor-progression $(P<0.01$, Table 1 and Fig. 1B, C). These data suggested that overexpression of ADNP associated with tumor-progression in patients treated with chemotherapy.

Table 1. Correlations of ADNP expression and clinicopathological features of bladder cancer patients

\begin{tabular}{|c|c|c|c|c|}
\hline \multirow[t]{2}{*}{ Characteristics } & \multirow[t]{2}{*}{$\mathrm{n}$} & \multicolumn{2}{|c|}{ ADNP expression } & \multirow{2}{*}{$P$-value } \\
\hline & & Low $(n=72)$ & High $(n=56)$ & \\
\hline \multicolumn{5}{|l|}{ Age (y) } \\
\hline$\geq 60$ & $72(56.3 \%)$ & 44 & 28 & 0.209 \\
\hline$<60$ & $56(43.8 \%)$ & 28 & 28 & \\
\hline \multicolumn{5}{|l|}{ Gender } \\
\hline Male & $61(47.7 \%)$ & 39 & 22 & 0.094 \\
\hline Female & $67(52.3 \%)$ & 33 & 34 & \\
\hline \multicolumn{5}{|l|}{ T stage } \\
\hline $\mathrm{T} 2$ & $85(66.4 \%)$ & 56 & 29 & 0.002 \\
\hline $\mathrm{T} 3, \mathrm{~T} 4$ & $43(33.6 \%)$ & 16 & 27 & \\
\hline \multicolumn{5}{|c|}{ Tumor multiplicity } \\
\hline Unifocal & $81(63.3 \%)$ & 52 & 29 & 0.017 \\
\hline Multifocal & $47(36.7 \%)$ & 20 & 27 & \\
\hline \multicolumn{5}{|l|}{ Tumor size $(\mathrm{cm})$} \\
\hline$<3$ & $76(59.4 \%)$ & 34 & 42 & 0.002 \\
\hline$\geq 3$ & $52(40.6 \%)$ & 38 & 14 & \\
\hline \multicolumn{5}{|l|}{ Vital states } \\
\hline non-progression & $73(57.0 \%)$ & 52 & 21 & $<0.001$ \\
\hline progression & $55(43.0 \%)$ & 20 & 35 & \\
\hline
\end{tabular}

\section{Patients with high ADNP expression were associated with poor prognosis}

In order to explore the relationship between ADNP expression and clinical parameters, we collected the clinical data of the $128 \mathrm{BC}$ patients and scored the degree of ADNP antibody staining. The clinical data of these paraffin-embedded BC specimens showed in Table 1. Kaplan-Meier survival curve showed that the no-progression was significantly shorter in patients with high expression of ADNP versus in the patients with low ADNP expression ( $\mathrm{P}<0.01$, Fig. 1D). Cox regression analysis also showed that ADNP was a risk prognostic factor for bladder tumor-progression after chemotherapy (Table 2). In summary, these data supported that ADNP upregulation in BC tissues was associated with 
poor prognosis in patients treated with chemotherapy.

Table 2. Univariate and multivariate Cox-regression analyses of prognostic factors in bladder cancer patients

\begin{tabular}{llllllll}
\hline Variables & HR & \multicolumn{2}{c}{ Univariate analysis } & \multirow{2}{*}{ HR } & & \multicolumn{2}{c}{ Multivariate analysis } \\
\cline { 3 - 4 } & & $95 \%$ CI & $p$-value & & & $95 \%$ CI & $p$-value \\
\hline Age & 1.129 & $0.655-1.129$ & 0.655 & - & - & - \\
Sex & 1.225 & $0.719-2.088$ & 0.455 & - & - & - \\
T stage & 3.823 & $2.232-6.549$ & $<0.001$ & 2.513 & $1.398-4.517$ & 0.002 \\
Tumor & 2.839 & $1.663-4.848$ & $<0.001$ & 1.923 & $1.073-3.447$ & 0.028 \\
multiplicity & & & & & & \\
Tumor size & 0.360 & $0.196-0.662$ & $<0.001$ & 0.484 & $0.256-0.917$ & 0.026 \\
ADNP & 3.362 & $1.936-5.838$ & $<0.001$ & 2.091 & $1.130-3.867$ & 0.019 \\
\hline
\end{tabular}

Abbreviations: HR: hazard ratio; CI: confidence interval.

\section{ADNP increased the cisplatin-resistance in BC cells}

In previous studies, we used western blotting to detect the basic expression of ADNP protein in four BC cell lines, in which it was low in 5637 and TCCSUP. By contrast, ADNP expression was much high in T24 and BIU87. Therefore, we performed a series of functional experiments using constructed stable ADNP knockdown and overexpressed cells to test whether ADNP contributes to the sensitivities of
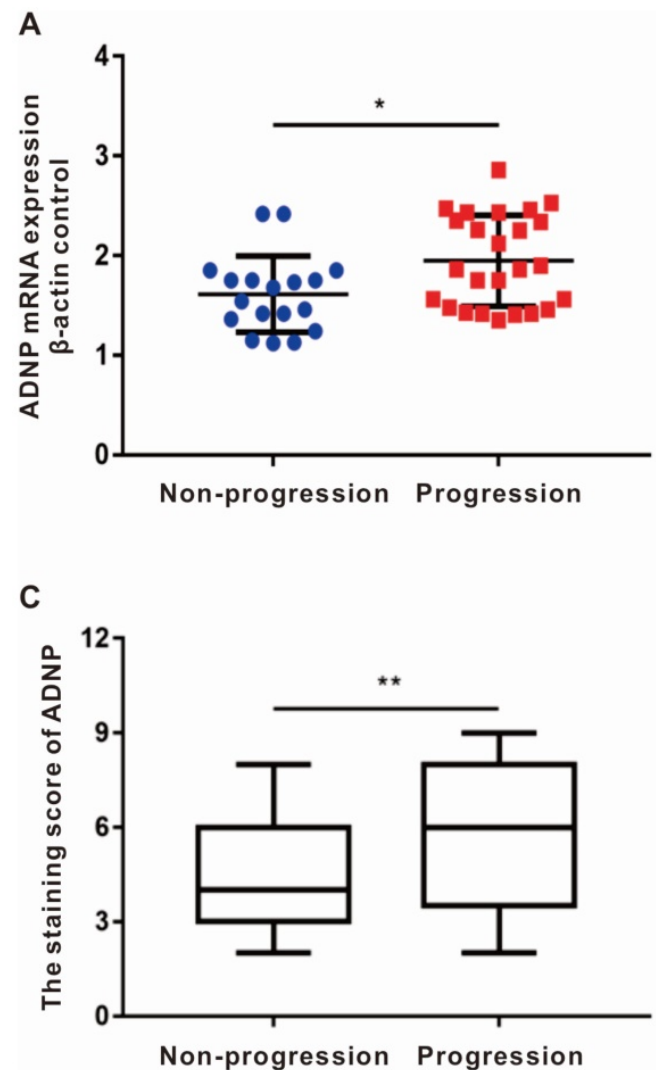

cisplatin in BC cells. We knocked down ADNP in T24 and Biu87 cells, respectively. CCK8 test showed that Knocking down ADNP reduced cell viability in both types of cells, and the promoting effect became stronger with the increase of cisplatin concentration. (Fig. 2A). Similarly, ADNP knockdown in the cells under cisplatin treatment also significantly reduced the average number of colonies in the colony formation experiment (Fig. 2C). Furthermore, we overexpressed ADNP in 5637 and TCCSUP cell lines, both CCK8 and colony formation analysis showed that ADNP overexpression significantly increased the cell viability and average colony number (Fig. 2B and 2D). These results indicated that ADNP may induce the resistance of $\mathrm{BC}$ cells to cisplatin.

\section{ADNP accelerated migration and promoted EMT in BC cells}

We performed wound healing experiment to explore the impact of ADNP on migration in BC cells. As shown in Fig. 3A, cells with ADNP knockdown exhibited delayed wound healing compared with NC group under cisplatin treatment. Overexpressed ADNP showed opposite results, suggesting that ADNP may promote the migration of $\mathrm{BC}$ cells under
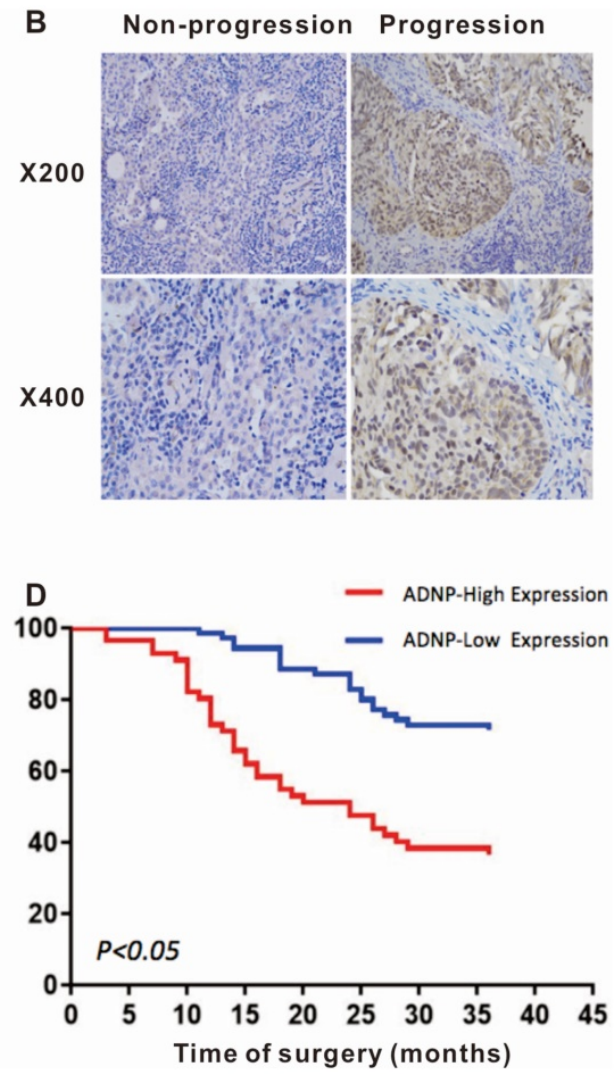

Figure 1. ADNP expression associates with tumor progression in patients after intravesical chemotherapy. A, ADNP mRNA in 43 bladder tumors, including 25 progression BC and 18 non-progression BC, were analyzed by using real-time quantitative PCR. Standardization was performed with $\beta$-actin as a control gene. B, Representative images of $A D N P$ protein expression in non-progression $B C$ and progression $B C$, the slides represent 50 microns. $\mathbf{C}$, Quantitative analysis of the average immunohistochemistry staining scores of ADNP in 73 cases non-progression BC tissue and 55 cases progression BC tissue after chemotherapy. D, Kaplan-Meier method analyzed the no progression survival in $128 \mathrm{BC}$ patients according to ADNP expression. * $\mathrm{P}<0.05$. 
cisplatin treatment. Interestingly, compared with NC group under cisplatin treatment, ADNPoverexpression up-regulated the expression of mesenchymal cell markers including N-Cadherin,
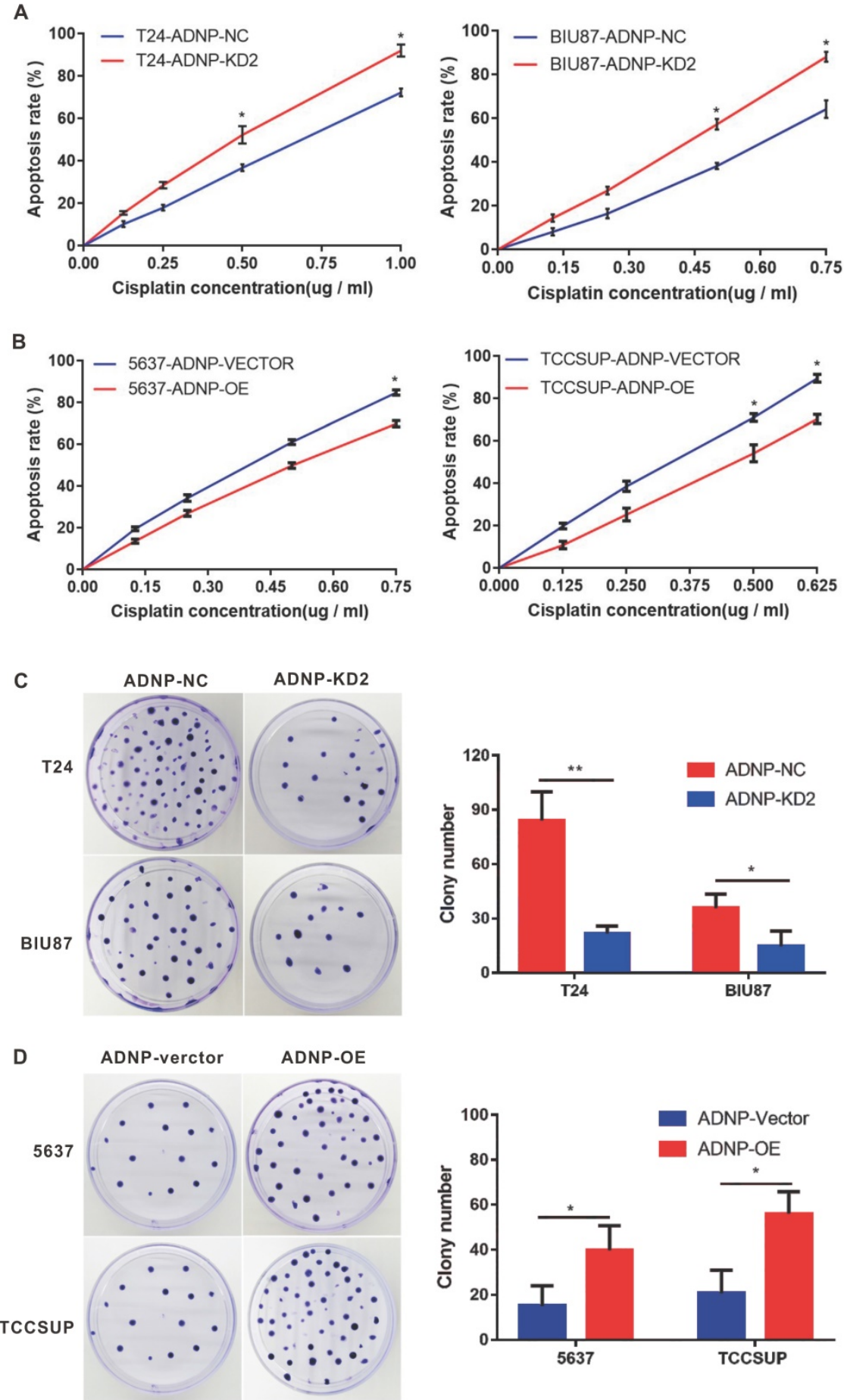

Figure 2. ADNP increases the cisplatin-resistance in BC cells. A, T24 and BIU87 cells were transfected with different ADNP-knockdown shRNA (KD-2) and negative control shRNA (NC) to establish the stable ADNP knockdown cell. CCK8 detected the apoptosis rate in BC cells under different concentrations of cisplatin. B, 5637 and TCCSUP cells transfected with ADNP overexpression plasmid (OE) and the empty vector ADNP (Vector) to establish the stable ADNP overexpressing cell. CCK8 detected the apoptosis rate of BC cells under different concentrations of cisplatin. C, Clone formation test to detect the growth of BC cells with the treatment of cisplatin between ADNP knockdown group and negative control group in T24 and BIU87. D, Clone formation test to detect the growth of BC cells treated with cisplatin between ADNP overexpressing group and blank control group in 5637 and TCCSUP. $* * \mathrm{P}<0.01, * \mathrm{P}<0.05$
Vimentin and snail as detected by western blot analysis, while the expression of E-cadherin, Catenin and Claudin-1 were down-regulated. By ADNP-knockdown showed the opposite (Fig. 3B). These data strongly suggested that ADNP induced EMT. Together, ADNP can increase the resistance of $\mathrm{BC}$ cells to cisplatin possibly by accelerating the migration and promoting EMT in BC cells.

\section{ADNP regulated the cisplatin-resistance of $B C$ in vivo}

In order to verify the experimental results in vitro, we constructed xenograft bladder tumor models using T24 bladder cells in NOD/SCID nude mice to evaluate the effect of ADNP on cisplatin response in vivo. As shown in Fig. 4A-C, cisplatin were associated with lower ability of tumor forming. ADNP knockdown inhibited tumor formation and higher drug response rate. Using immunohistochemistry on isolated nude mouse tumor tissues, the results confirmed that ADNP knockdown significantly downregulated the expression of ADNP, N-Cadherin, and Vimentin after cisplatin intervention, while E-Cadherin expression was the opposite (Fig. 4D). These results in vivo suggested that ADNP may play an important role in the development of cisplatin resistance in BC.

\section{ADNP activated TGF- $\beta / S m a d$ signaling pathway}

To further study the possible molecular mechanism why ADNP regulated EMT and cisplatinresistance, we treated the $\mathrm{BC}$ cells with cisplatin $(0.125 \mathrm{ug} / \mathrm{ml}, 48 \mathrm{~h})$ and used western bolt to explore the protein expression of the key proteins in the TGF- $\beta /$ Smad, which is classic signaling pathway in EMT processes. As shown in the experiments with ADNP knockdown in T24 and BIU87 BC cells, 
expression of TGF- $\beta$, TGF- $\beta$ R1, Smad2, Smad3, p-Smad2 and p-Smad3 was significantly decreased, while overexpression of ADNP in 5637 and TCCSUP BC cells produced the opposite effects (Fig. 5). These results suggested that ADNP activated the TGF- $\beta$ /Smad signaling pathway. ADNP promoted EMT and induced cisplatin resistance in BC cells, which may be related to TGF- $\beta /$ Smad signaling pathway.
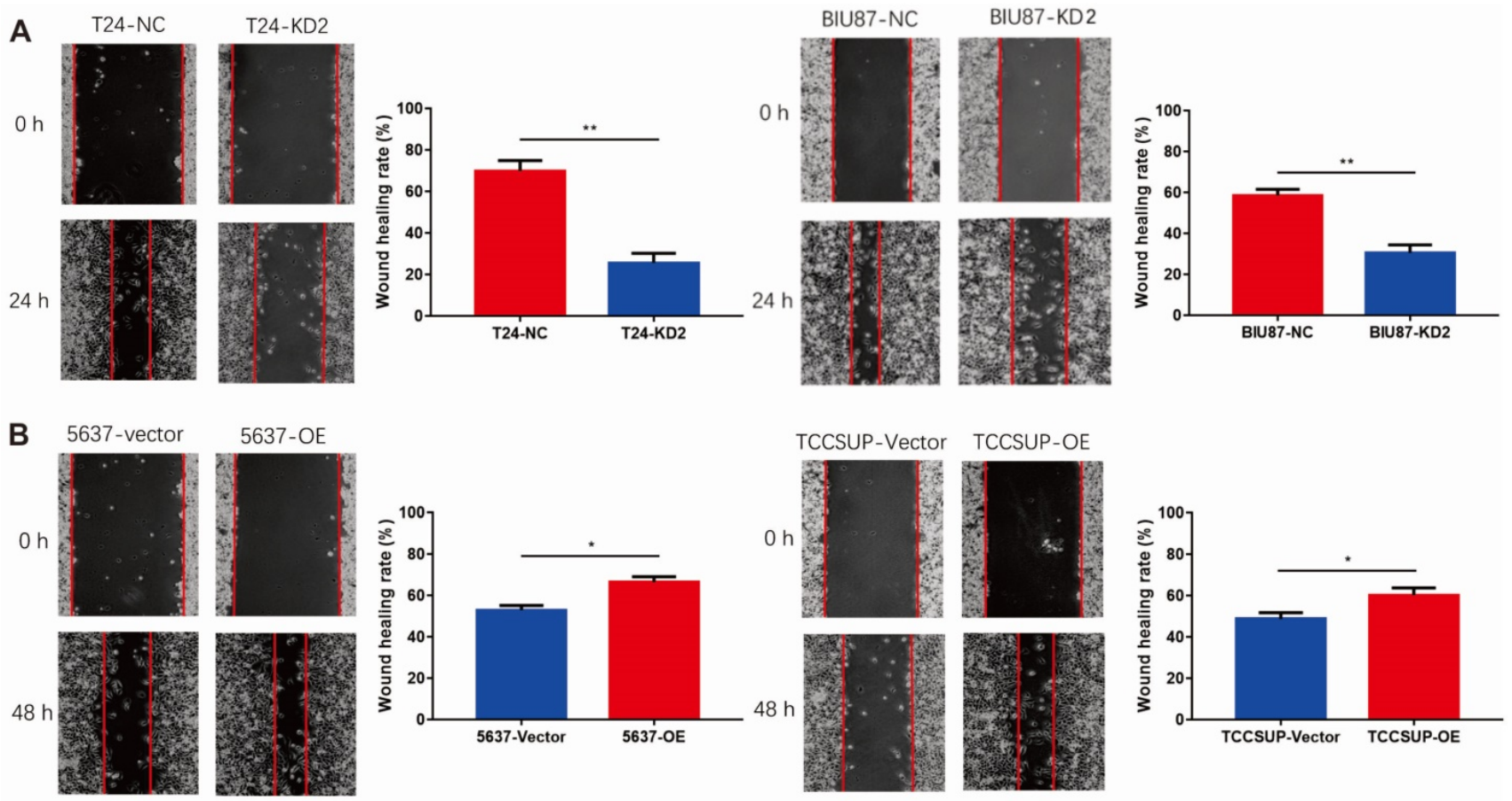
Our results, for the first time, indicate that ADNP expression was higher in progression $\mathrm{BC}$ compared to non-progression BC. Patients with high ADNP expression in $\mathrm{BC}$ had significantly shorter survival after chemotherapy. Here we provided the potential evidences that ADNP induced cisplatin-resistance.

\section{Discussion}

In this study, we analyzed the relationship between ADNP and cisplatin-resistance in human BC.

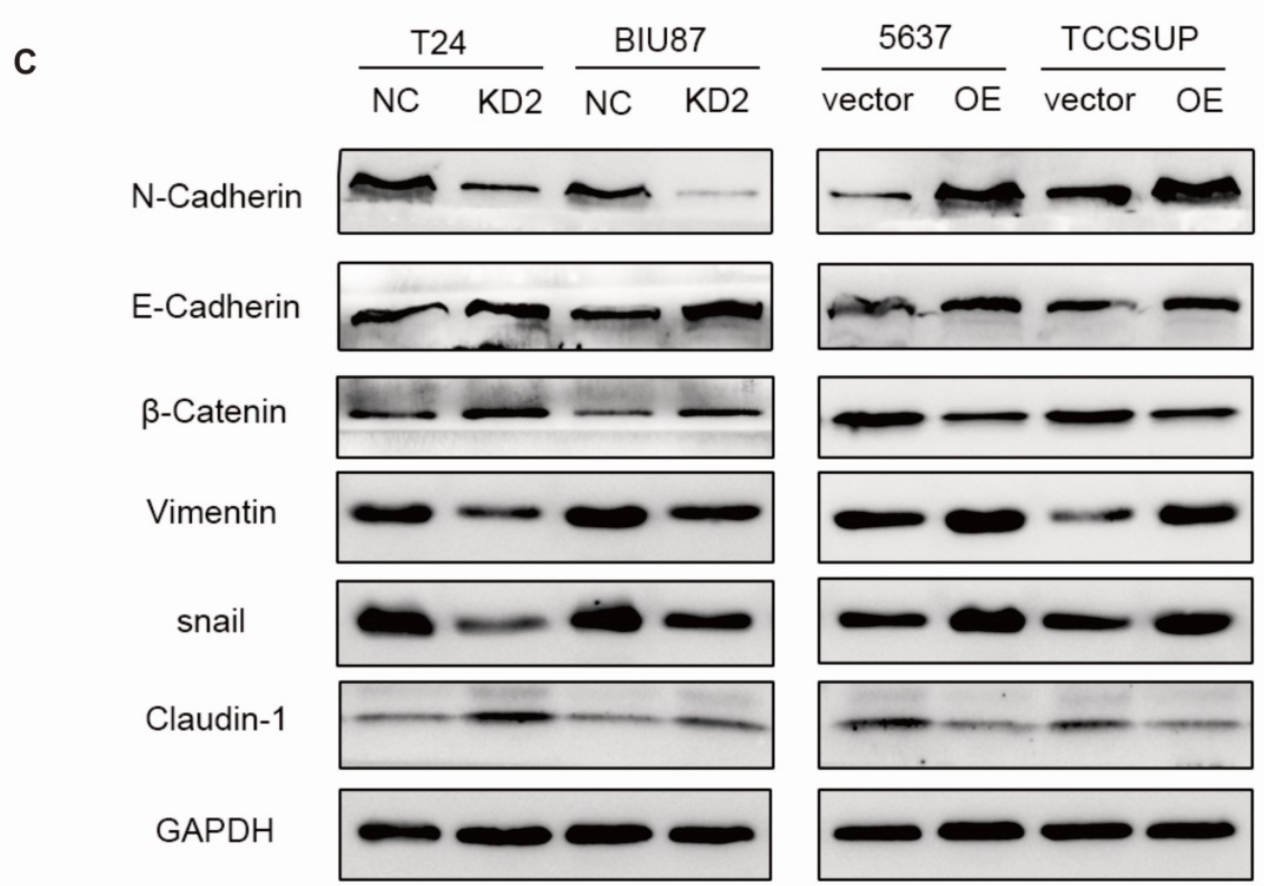

Figure 3. ADNP accelerates migration of BC cells. A, Wound healing assay was used for validating the effect of ADNP on migration in BC. B, Western blot was used to detect the expression of N-Cadherin, E-Cadherin, $\beta$-Catenin, Vimentin, snail, and Claudin- 1 in the different groups. $* * P<0.01, * P<0.05$. 
A

T24

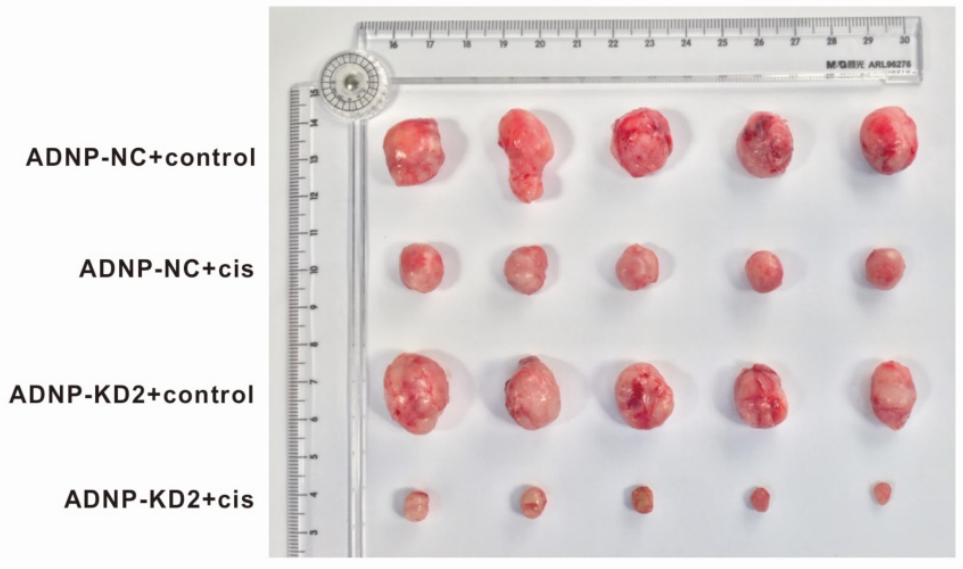

B

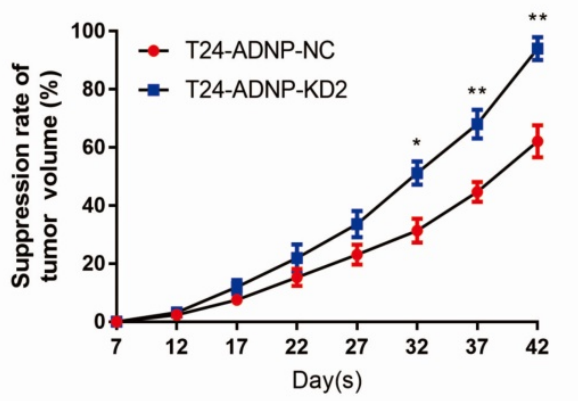

C

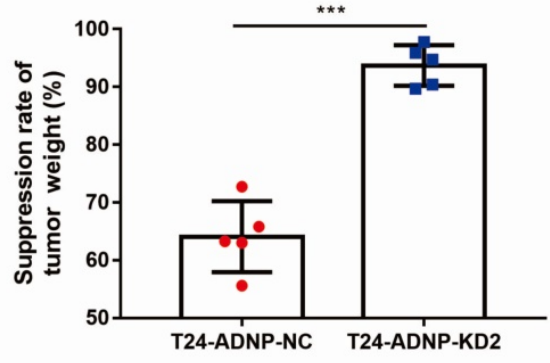

D

ANDP

$\mathrm{N}$-Cadherin
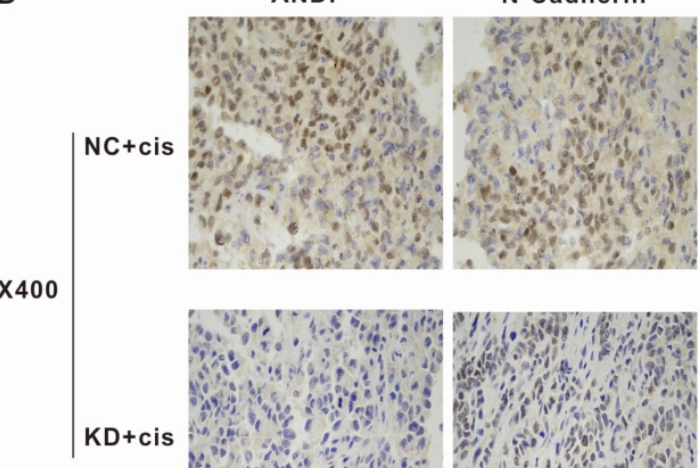

E-Cadherin

Vimentin
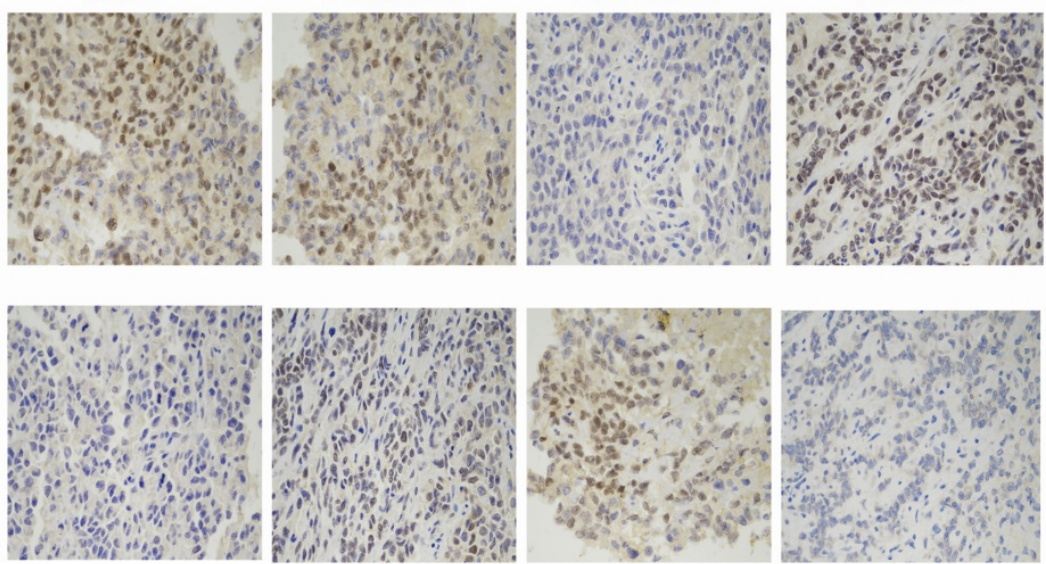

Figure 4. ADNP regulates the cisplatin-resistance in BC in vivo. A, 4 groups image of tumors dissected from NOD/SCID nude mice. B, Tumors volume was measured from the 7th day after tumor implantation, the continuous growth curve was drawn. C, Comparison of the average tumor resection weight of the control group and the knockdown group with the treatment of cisplatin. D, Immunohistochemical detection of representative images of ADNP, N-Cadherin, E-Cadherin, and Vimentin in two groups of nude mice. $* * * \mathrm{P}<0.001, * * \mathrm{P}<0.01, * \mathrm{P}<0.05$

ADNP encodes a protein that is involved in the development of the nervous system in embryos [33]. Besides, ADNP gene is also closely linked with tumor cells proliferation, invasion as well as migration [15]. Wound healing experiments showed that ADNP promoted cell migration $[15,34]$. A recent study showed that the expression of ADNP enhanced the adaptive ability of malignant peripheral nerve sheath tumors to survive in a malignant environment $[16,35]$ [16]. The expression of ADNP in bladder was related to age and sex, and is higher in young men [36]. Here we found that the high expression of ADNP leaded to unfavorable prognosis in patients treated with chemotherapy. Thus, we hypothesized that ADNP may promote cells proliferation and cell migration, thereby increasing the chemoresistance in BC. In this study, we verified our hypotheses by using in vitro experiments. Our results revealed that ADNP knockdown in BC cell lines significantly reduced cells proliferation and cell migration under cisplatin, while ADNP upregulation showed the opposite results. These results supported that ADNP had an effect on BC chemoresistance. 


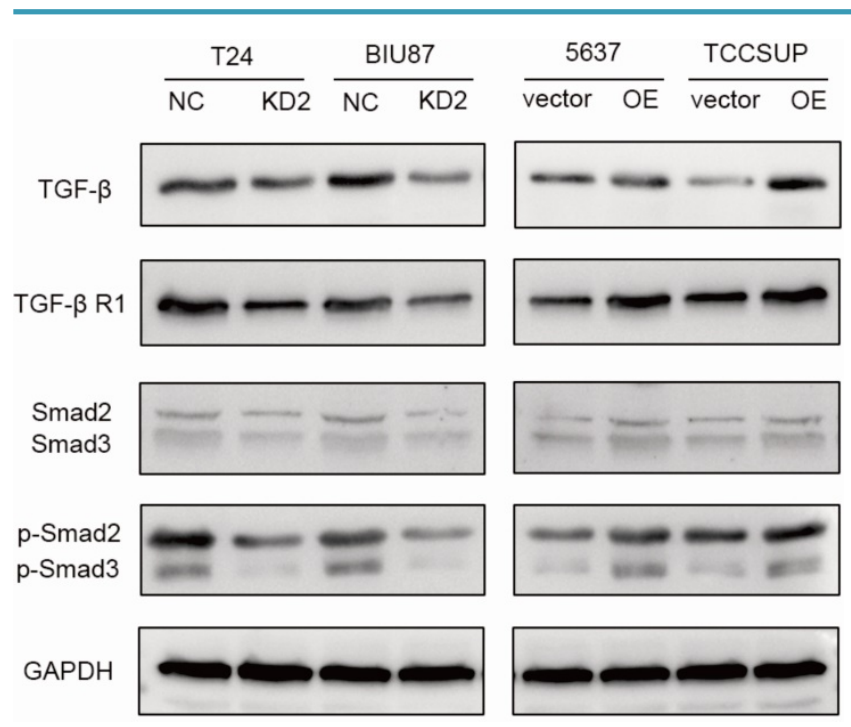

Figure 5. ADNP activates TGF- $\beta$ pathway in BC cells. BC cells were treated with cisplatin $(0.125 \mu \mathrm{g} / \mathrm{ml}, 48 \mathrm{~h})$. The expression of the key proteins in the TGF- $\beta /$ Smad, including TGF- $\beta$, TGF- $\beta R 1$, Smad2/Smad3, $p-S m a d 2 / p-S m a d 3$ were detected in $B C$ cells by using western blot.

EMT is considered to be closely related to tumor progression and chemotherapy resistance [10]. Gene expression alterations during EMT processes resulted in many phenotypic changes such as changes in cell morphology, loss of adhesion, and acquisition of stem cell-like features [37]. In most cases, the loss of E-cadherin is a hallmark of EMT. For instance, MCF-7 breast cancer cell line resistant to adriamycin and the ZR-75-B breast cancer cell line resistant to vincristine exhibited EMT and vimentin high expression, while E-cadherin, formation of desmosomes and tight junction expression were reduced [38]. In this study, we used immunoblotting to analyze the expression of EMT-related biomarkers in BC cell lines. ADNP upregulation increased the expression of mesenchymal biomarkers like $\mathrm{N}$-cadherin, vimentin as well as snail, whereas it reduced the expression of epithelial proteins like E-cadherin, $\beta$-catenin and Claudin-1, which consist with previous founding. By contrast, ADNP downregulation showed the opposite results, indicating that ADNP promoted the EMT in BC cells. A close relationship between ADNP-induced EMT and cisplatin-resistance were also established on mice models. Subcutaneous xenotransplantation was used to establish a model in nude mice. The results showed that cisplatin were associated with lower ability of tumor forming. ADNP knockdown inhibited tumor formation and higher drug response rate. ADNP knockdown significantly down-regulated the expression of ADNP, N-Cadherin, and Vimentin after cisplatin intervention, while E-Cadherin expression was the opposite. These data were consistent the research on lung cancer. The growth rate of primary tumor in mice was reduced by $60 \%$ by treatment with the cyclophosphamide (a chemotherapeutic drug).
However, as compared with epithelial-type cancer cells, EMT-positive cells in primary tumors were resistant to drug induced cell apoptosis. The number of cells were without significantly decreasing under chemotherapy [39]. Above data revealed that EMT might have contribution on drug resistance in BC.

Transforming growth factor beta (TGF- $\beta$ ), which played an important role in cell proliferation, differentiation, embryogenesis and morphogenesis, is thought to be a main pathway of the EMT process in cancer [40]. In classic TGF- $\beta /$ Smad signaling, TGF- $\beta$ ligand binded to the type II TGF- $\beta$ receptor (T $\beta$ RII) on the cell membrane to form the TGF- $\beta /$ T $\beta$ RII complex to activate the type I TGF- $\beta$ receptor (T $\beta R I)[41,42]$. Phosphorylation of T $\beta R I$ activated phosphorylation of Smad 2 and Smad3 in cytoplasm [43]. Phosphorylation of Smad2/3 caused a trimer formation between phosphorylated Smad2/3 and Smad 4 [44]. The receptor activated Smad complex activated or inhibited its target gene promoter, thereby promoting EMT [45]. Studies showed that long-term exposure to EGFR-TKIs activated TGF- $\beta /$ Smad signaling and promoted the EMT phenotype in non-small cell lung cancer [9]. In line with this, we knocked down ADNP in T24 and BIU87 BC cells and found the expression of TGF $\beta$, TGF- $\beta$ RI and $p-S m a d 2 / 3$ were decreased, in contrast, overexpression of ADNP in 5637 and TCCSUP BC cells showed opposite results, indicating that ADNP promoted cell migration and EMT, thereby inducing cisplatin resistance, which may be related to TGF- $\beta /$ Smad signaling pathway. TGF- $\beta$ belongs to the TGF- $\beta$ superfamily, which regulates cell growth and differentiation, and can transform the phenotype of normal fibroblasts. The TGF- $\beta$ pathway was affected by changes in epithelial cell adhesion molecules [46]. Previous studies have shown that cell growth and migration were regulated by cell adhesion (such as cadherins, selectins), while ADNP promoted cell adhesion [47]. Therefore, we speculated that ADNP may affect the activation of TGF- $\beta$ signaling pathway by regulating cell adhesion, thereby promoting EMT and increasing the resistance of $\mathrm{BC}$ cells to cisplatin, which is the content of our next study.

In summary, our study showed that ADNP was significantly upregulated and was closely related to unfavorable prognosis in progression BC after chemotherapy. ADNP promoted cell migration and EMT, thereby inducing cisplatin resistance, which may be related to TGF- $\beta /$ Smad signaling pathway. Our results provided a mechanistic explanation for the involvement of ADNP in BC progression under chemotherapy. ADNP may be a novel molecular target for predicting prognosis and cancer therapy in patients with $\mathrm{BC}$ after chemotherapy. 


\section{Acknowledgements}

This study was funded by the Key Research and Development Projects of Hunan Province (grant number 2018SK2125) and China national cancer climbing fund (grant number NCC201818A55).

\section{Ethics statement}

Written informed consent was obtained from all patients, and care and use of animals in all experiments complied with institutional and national recommendations. This study was approved by the ethics committees of the Affiliated Cancer Hospital of Xiangya School of Medicine, Central South University, and Hunan Cancer Hospital.

\section{Data availability}

All datasets generated for this study are included in the manuscript.

\section{Competing Interests}

The authors have declared that no competing interest exists.

\section{References}

1. Bhanvadia S K. Bladder Cancer Survivorship. Curr Urol Rep. 2018; 19: 111.

2. Chen J, Li Y, Li Z, et al. LncRNA MST1P2/miR-133b axis affects the chemoresistance of bladder cancer to cisplatin-based therapy via Sirt1/p53 signaling. J Biochem Mol Toxicol. 2020; 34: e22452.

3. Kitamura T, Suzuki M, Nishimatsu H, et al. Final report on low-dose estramustine phosphate (EMP) monotherapy and very low-dose EMP therapy combined with LH-RH agonist for previously untreated advanced prostate cancer. Aktuelle Urol. 2010; 41 (Suppl 1): S34-S40.

4. Li Y, Li G, Guo X, et al. Non-coding RNA in bladder cancer. Cancer Lett. 2020; 485: 38-44

5. Jiménez-Garduño A M, Mendoza-Rodríguez M G, Urrutia-Cabrera D, et al. IL-1beta induced methylation of the estrogen receptor ERalpha gene correlates with EMT and chemoresistance in breast cancer cells. Biochem Biophys Res Commun. 2017; 490: 780-785.

6. Li H, Li J, Chen L, et al. HERC3-Mediated SMAD7 Ubiquitination Degradation Promotes Autophagy-Induced EMT and Chemoresistance in Glioblastoma. Clin Cancer Res. 2019; 25: 3602-3616.

7. Yang Y, Yao J H, Du Q Y, et al. Connexin 32 downregulation is critical for chemoresistance in oxaliplatin-resistant HCC cells associated with EMT. Cancer Manag Res. 2019; 11: 5133-5146.

8. Zeng D, Liang Y K, Xiao Y S, et al. Inhibition of Notch1 reverses EMT and chemoresistance to cisplatin via direct downregulation of MCAM in triple-negative breast cancer cells. Int J Cancer. 2020.

9. Soucheray M, Capelletti M, Pulido I, et al. Intratumoral Heterogeneity in EGFR-Mutant NSCLC Results in Divergent Resistance Mechanisms in Response to EGFR Tyrosine Kinase Inhibition. Cancer Res. 2015; 75: 4372-83.

10. Kotiyal S, Bhattacharya S. Breast cancer stem cells, EMT and therapeutic targets. Biochem Biophys Res Commun. 2014; 453: 112-6.

11. Hashmi F, Liu M, Shen S, et al. Phospholipase C gamma mediates endogenous brain-derived neurotrophic factor - regulated calcitonin gene-related peptide expression in colitis - induced visceral pain. Mol Pain. 2016; 12.

12. Karagoz K, Mehta G A, Khella C A, et al. Integrative proteogenomic analyses of human tumours identifies ADNP as a novel oncogenic mediator of cell cycle progression in high-grade serous ovarian cancer with poor prognosis. EBioMedicine. 2019; 50: 191-202.

13. Zamostiano R, Pinhasov A, Gelber E, et al. Cloning and characterization of the human activity-dependent neuroprotective protein. J Biol Chem. 2001; 276: 708-14.

14. Gozes I, Yeheskel A, Pasmanik-Chor M. Activity-dependent neuroprotective protein (ADNP): a case study for highly conserved chordata-specific genes shaping the brain and mutated in cancer. J Alzheimers Dis. 2015; 45: 57-73.

15. Blaj C, Bringmann A, Schmidt E M, et al. ADNP Is a Therapeutically Inducible Repressor of WNT Signaling in Colorectal Cancer. Clin Cancer Res. 2017; 23: 2769-2780

16. Castorina A, Giunta S, Scuderi S, et al. Involvement of PACAP/ADNP signaling in the resistance to cell death in malignant peripheral nerve sheath tumor (MPNST) cells. J Mol Neurosci. 2012; 48: 674-83.
17. Zhu S, Xu Z, Zeng Y, et al. ADNP Upregulation Promotes Bladder Cancer Cell Proliferation via the AKT Pathway. Front Oncol. 2020; 10: 491129.

18. Gupta S, Chaudhary S, Bubber P, et al. Epidemiology and genetic diversity of group A rotavirus in acute diarrhea patients in pre-vaccination era in Himachal Pradesh, India. Vaccine. 2019; 37: 5350-5356.

19. Shu-Jun X, Kang W, Min-Hong Z, et al. Comparison of three different methods for isolating RNA from Oncomelania hupensis. Zhongguo Xue Xi Chong Bing Fang Zhi Za Zhi. 2017; 29: 334-337.

20. Wang $S \mathrm{Q}, \mathrm{Xu} \mathrm{B}, \mathrm{Liu}$ J, et al. Effect of RNA interference targeting Schistosoma japonicum aldose reductase gene. Zhongguo Ji Sheng Chong Xue Yu Ji Sheng Chong Bing Za Zhi. 2014; 32: 17-21.

21. Li T S C, Yawata T, Honke K. Efficient siRNA delivery and tumor accumulation mediated by ionically cross-linked folic acid-poly(ethylene glycol)-chitosan oligosaccharide lactate nanoparticles: for the potential targeted ovarian cancer gene therapy. Eur J Pharm Sci. 2014; 52: 48-61.

22. Kang J F, Hu H H, Chen R. In vitro observation on the apoptosis induced by $\mathrm{H} 2 \mathrm{O} 2$ in protoscolex of Echinococcus granulosus]. Zhongguo Ji Sheng Chong Xue Yu Ji Sheng Chong Bing Za Zhi. 2008; 26: 332-7.

23. Nguyen H M, Sako K, Matsui A, et al. Ethanol Enhances High-Salinity Stress Tolerance by Detoxifying Reactive Oxygen Species in Arabidopsis thaliana and Rice. Front Plant Sci. 2017; 8: 1001

24. Wang L, Ouyang F, Liu X, et al. Overexpressed CISD2 has prognostic value in human gastric cancer and promotes gastric cancer cell proliferation and tumorigenesis via AKT signaling pathway. Oncotarget. 2016; 7: 3791-805.

25. Chen $Y$, Jiang H, Cao J. Influence of Photodynamic Therapy on Apoptosis and Invasion of Human Cholangiocarcinoma QBC939 Cell Line. Chin Med Sci J. 2015; 30: 252-9.

26. Gong S, Tao Z, Liu X, et al. An underlying prognosis predictor of hepatocellular carcinoma: Oncoprotein 18. Biomed Rep. 2014; 2: 85-88.

27. Wilson C, Lukowicz R, Merchant S, et al. Quantitative and Qualitative Assessment Methods for Biofilm Growth: A Mini-review. Res Rev J Eng Technol. 2017; 6 .

28. Guzman C, Bagga M, Kaur A, et al. ColonyArea: an ImageJ plugin to automatically quantify colony formation in clonogenic assays. PLoS One. 2014; 9: e92444.

29. Arranz-Valsero I, Soriano-Romaní L, García-Posadas L, et al. IL-6 as a corneal wound healing mediator in an in vitro scratch assay. Exp Eye Res. 2014; 125: 183-92.

30. Perfetto B, Stellavato A, Melito A, et al. A time-lapse approach to examine chromium and nickel effects on wound healing in vitro. J Immunotoxicol. 2012; 9: 392-400.

31. Wang B, Chen Q, Cao Y, et al. LGR5 Is a Gastric Cancer Stem Cell Marker Associated with Stemness and the EMT Signature Genes NANOG, NANOGP8, PRRX1, TWIST1, and BMI1. PLoS One. 2016; 11: e0168904.

32. Lehmann $C$, Friess $T$, Birzele $F$, et al. Superior anti-tumor activity of the MDM2 antagonist idasanutlin and the Bcl-2 inhibitor venetoclax in p53 wild-type acute myeloid leukemia models. J Hematol Oncol. 2016; 9: 50.

33. Furman S, Hill J M, Vulih I, et al. Sexual dimorphism of activity-dependent neuroprotective protein in the mouse arcuate nucleus. Neurosci Lett. 2005; 373: 73-8.

34. Mollinedo P, Kapitansky O, Gonzalez-Lamuño D, et al. Cellular and animal models of skin alterations in the autism-related ADNP syndrome. Sci Rep. 2019; 9: 736

35. Suzuki M, Liu M, Kurosaki T, et al. Association of rs6983561 polymorphism at $8 \mathrm{q} 24$ with prostate cancer mortality in a Japanese population. Clin Genitourin Cancer. 2011; 9: 46-52.

36. Kapitansky O, Sragovich S, Jaljuli I, et al. Age and Sex-Dependent ADNP Regulation of Muscle Gene Expression Is Correlated with Motor Behavior: Possible Feedback Mechanism with PACAP. Int J Mol Sci. 2020; 21.

37. Chen T, You Y, Jiang H, et al. Epithelial-mesenchymal transition (EMT): A biological process in the development, stem cell differentiation, and tumorigenesis. J Cell Physiol. 2017; 232: 3261-3272.

38. Sommers C L, Heckford S E, Skerker J M, et al. Loss of epithelial markers and acquisition of vimentin expression in adriamycin- and vinblastine-resistant human breast cancer cell lines. Cancer Res. 1992; 52: 5190-7.

39. Kuczynski E A, Krueger J, Chow A, et al. Impact of Chemical-Induced Mutational Load Increase on Immune Checkpoint Therapy in Poorly Responsive Murine Tumors. Mol Cancer Ther. 2018; 17: 869-882.

40. David C J, Huang Y H, Chen M, et al. TGF- $\beta$ Tumor Suppression through a Lethal EMT. Cell. 2016; 164: 1015-30.

41. Zhang J, Wang Y, Weng $\mathrm{H}$, et al. Management of non-muscle-invasive bladder cancer: quality of clinical practice guidelines and variations in recommendations. BMC Cancer. 2019; 19: 1054.

42. Deng G, Chen L, Zhang Y, et al. Fucosyltransferase 2 induced epithelialmesenchymal transition via TGF-beta/Smad signaling pathway in lung adenocarcinaoma. Exp Cell Res. 2018; 370: 613-622.

43. Chen H, Cai J, Wang J, et al. Targeting Nestin(+) hepatic stellate cells ameliorates liver fibrosis by facilitating T $\beta$ RI degradation. J Hepatol. 2020.

44. Martin-Malpartida P, Batet M, Kaczmarska Z, et al. Structural basis for genome wide recognition of 5-bp GC motifs by SMAD transcription factors. Nat Commun. 2017; 8: 2070.

45. Sakai S, Ohhata T, Kitagawa K, et al. Long Noncoding RNA ELIT-1 Acts as a Smad3 Cofactor to Facilitate TGF $\beta /$ Smad Signaling and Promote EpithelialMesenchymal Transition. Cancer Res. 2019; 79: 2821-2838. 
46. Martin M E, Reaves D K, Jeffcoat B, et al. Silver nanoparticles alter epithelial basement membrane integrity, cell adhesion molecule expression, and TGF- $\beta 1$ secretion. Nanomedicine. 2019; 21: 102070.

47. Greenberg D A. Linking acquired neurodevelopmental disorders to defects in cell adhesion. Proc Natl Acad Sci U S A. 2003; 100: 8043-4. 